Effect of lifestyle counselling on health-related quality of life in women at high risk for gestational diabetes

\title{
Sahrakorpi, Niina
}

2019-06

Sahrakorpi , N , Rönö , K , Koivusalo , S B , Stach-Lempinen , B , Eriksson , J G \& Roine , R $P 2019$, ' Effect of lifestyle counselling on health-related quality of life in women at high risk for gestational diabetes ', European Journal of Public Health , vol. 29 , no. 3 , pp. 408-412 . https://doi.org/10.1093/e

http://hdl.handle.net/10138/306695

https://doi.org/10.1093/eurpub/cky248

publishedVersion

Downloaded from Helda, University of Helsinki institutional repository.

This is an electronic reprint of the original article.

This reprint may differ from the original in pagination and typographic detail.

Please cite the original version. 
33 Bourgeault IL, Demers C, Donovan S. A report commissioned by Peel Public Health. In public health workforce development models: literature scan, review \& synthesis. 2009. Available at: http://www.peel.ca/health/resources/pdf/Ivy-BourgeaultWorkforce\%20Development.pdf (May 2018, date last accessed).

34 Hilliard TM, Boulton ML. Public health workforce research in review: a 25-year retrospective. Am J Prev Med 2012;42:S17-28.

35 Paccaud F, Weihofen A, Frank M. Public Health Workforce in Switzerland: are public health workers lacking? Int J Public Health 2013;58:799-800.

36 Ishikawa K. Guide to Quality Control. Industrial Engineering and Technology Series. Tokyo: Quality Resources, 1986.

37 Minnesota Department of Public Health (MDH). Fishbone Diagram. 2017. Available at: http://www.health.state.mn.us/divs/opi/qi/toolbox/fishbone.html (May 2018, date last accessed).
38 Malgieri A, Michelutti P, Van Hoegaerden M, Joint Action Healh Workforce Programming \& Forecasting. Handbook on Health Workforce Planning Methodologies across EU Countries. Bratislava: The Ministry of Health of the Slovak Republic, 2015. Available at: http://healthworkforce.eu/wp-content/uploads/2015/11/150306_WP5_ D052-Handbook-on-HWF-Planning-Methodologies-across-EU-Countries_Release1_Final-version.pdf (May 2018, date last accessed).

39 Ministry of Health New Zealand. The Credentialling Framework for New Zealand Health Professionals. 2010. Available at: http://www.cmc.org.nz/media/41302/moh resource_on_credentialling.pdf (May 2018, date last accessed).

40 American Public Health Association. Ethics. 2018. Available at: https://www. apha.org/apha-communities/member-sections/ethics (May 2018, date last accessed).

The European Journal of Public Health, Vol. 29, No. 3, 408-412

(C) The Author(s) 2018. Published by Oxford University Press on behalf of the European Public Health Association. All rights reserved. doi:10.1093/eurpub/cky248 Advance Access published on 29 November 2018

\title{
Effect of lifestyle counselling on health-related quality of life in women at high risk for gestational diabetes
}

\author{
Niina Sahrakorpi $\mathbb{D}^{1,2}$, Kristiina Rönö ${ }^{1}$, Saila B. Koivusalo ${ }^{1}$, Beata Stach-Lempinen ${ }^{3}$, \\ Johan G. Eriksson ${ }^{4,5}$, Risto P. Roine ${ }^{6,7}$
}

1 Department of Obstetrics and Gynecology, University of Helsinki and Helsinki University Hospital, Women's Hospital, Helsinki, Finland

2 City of Vantaa, Preventive Medical Services, Communal Maternity Clinic, Vantaa, Finland

3 Department of Obstetrics and Gynecology, South Karelia Central Hospital, Lappeenranta, Finland

4 Department of General Practice and Primary Health Care, University of Helsinki and Helsinki University Hospital, Helsinki, Finland

5 Folkhälsan Research Center, University of Helsinki, Helsinki, Finland

6 Department of Health and Social Management, Research Centre for Comparative Effectiveness and Patient Safety, University of Eastern Finland and Kuopio University Hospital, Kuopio, Finland

7 Group Administration, University of Helsinki and Helsinki University Hospital, Helsinki, Finland

Correspondence: Niina Sahrakorpi, City of Vantaa, Preventive Medical Services, Communal Maternity Clinic, c/o Niittyluhdantie 4, 00660 Helsinki, Finland, Tel: +358 (0) 503235172, e-mail: niina.sahrakorpi@vantaa.fi

\begin{abstract}
Background: The incidence of gestational diabetes (GDM) is increasing and interventions to curb the detrimental effects of GDM are needed. We have previously reported that a combined diet and physical activity intervention has the potential to reduce GDM among high-risk women. It is also important to know whether the intervention affects health-related quality of life (HRQoL). Methods: A total of 378 women at high risk for GDM were randomized into an intervention (lifestyle counselling four times during pregnancy, $n=192$ ), or a control group $(n=186)$ before 20 gestational weeks. HRQoL was assessed with the 15D-instrument six times: once during each trimester and at six weeks, six months and 12 months postpartum. Results: In this study population, the cumulative incidence of GDM was similar in the intervention and the control group (45.7 vs. $44.5 \%$ ). There was no difference between the 15D scores of the control and intervention groups at any of the time points. Conclusions: Combined diet and physical activity intervention did not provide HRQoL benefits in the study. A high prevalence of GDM in both study groups may have confounded the effect of the intervention.
\end{abstract}

\section{Introduction}

G estational diabetes (GDM), defined as a glucose metabolism dis3 turbance that is first detected during pregnancy, is increasing globally. As GDM is associated with short- and long-term complications for both the mother and the child, it is desirable to find strategies to prevent GDM. Studies assessing the efficacy of lifestyle interventions (mixed diet and physical activity) in preventing GDM and associated pregnancy complications have however been heterogeneous, and the results somewhat inconclusive with some positive results. ${ }^{1-3}$

WHO defines quality of life (QoL) as 'individuals' perceptions of their position in life in the context of the culture and value systems in which they live and in relation to their goals, expectations, standards, and concerns'. ${ }^{4}$ Health-related quality of life (HRQoL) can be defined as 'The (mainly) subjective quality of life of the individual in relation to health or in response to the onset of disease' including the individual's physical and mental health perceptions together with health risks and conditions, functional status, social support, socioeconomic status and also the factors of health care. ${ }^{4,5}$ As such, compared to clinical parameters, HRQoL introduces a more holistic and subjective, yet quantifiable measure to estimate individual's functional health-status also in therapeutic and research settings.

Measures of HRQoL can be divided into two groups according to their content, either generic or specific. Generic measures produce health profiles and utility measures, as specific measures focus on the 
health domains or functional aspects of special interest in a certain disease. $^{5}$

Generic utility data is a pre-requisite for cost-utility analyses, which combine the length and QoL into a single measurement of quality adjusted life years that are related to the costs of the intervention. 6

We have previously shown that HRQoL deteriorates towards the third trimester of pregnancy and returns to baseline levels soon after delivery. ${ }^{8}$ Pre-pregnancy obesity and excessive gestational weight gain also have a negative impact on the peripartal HRQoL during pregnancy ${ }^{9}$ even in the absence of pregnancy-related complications. ${ }^{8}$

Most previous studies having employed various HRQoL instruments (15D, SF-36, visual analogue component of EQ-5D-3 L) suggest that the diagnosis of GDM per se does not seem to affect women's perception of their health-status or HRQoL during pregnancy or postpartum. ${ }^{8,10-12}$ However, women with GDM have shown to value their general health-status (measured with SF-36) in the third trimester lower compared to women without GDM. ${ }^{13} \mathrm{~A}$ recent review suggested that decreased QoL may be possible to improve through diabetes-related self-management interventions (diet, exercise, self-monitoring, insulin control). ${ }^{14}$

The knowledge of the effects of general lifestyle interventions on HRQoL during normal pregnancy is scarce. Some studies have suggested that general physical activity of healthy pregnant women has positive effects on perinatal HRQoL, ${ }^{15,16}$ whereas others have not found an effect. ${ }^{17,18}$

There is no evidence that lifestyle interventions aiming to reduce GDM have an effect on HRQoL. ${ }^{19-24}$ Recent systematic reviews have looked into the effects of treating GDM with exercise, diet and combined lifestyle interventions, and concluded that the shortand long-term cost-effectiveness of the interventions remain unclear. $^{25,26}$

This study is a secondary analysis of the Finnish Gestational Diabetes Prevention Study (RADIEL) which aimed to prevent GDM and its complications in high-risk women with a combined physical activity and dietary intervention. The RADIEL-intervention initiated during early pregnancy succeeded in reducing GDM incidence by $36 \%$ among women with normal glucose tolerance at baseline. $^{14}$

The aim of this study is (i) to evaluate the effect of lifestyle counselling on HRQoL during and after pregnancy within the study population recruited in early pregnancy (including also women with early GDM) and (ii) to provide HRQoL data that can be used as a basis for the cost-utility analysis of the RADIELstudy in the future.

\section{Methods}

The RADIEL trial was a multicentre lifestyle intervention trial conducted between 2008 and 2014 in Finland. ${ }^{27}$ The main aim of the trial was to evaluate the efficacy of a combined diet and physical activity intervention in prevention of GDM and related adverse outcomes, in a setting applicable to primary health care. The study sites were the Helsinki University Hospital ( $\mathrm{HUH}$, including the Women's Hospital and the Kätilöopisto Maternity Hospital in Helsinki, and the Jorvi Hospital in Espoo) and the South-Karelia Central Hospital (SKCH) in Lappeenranta.

The participants of this trial had to be (i) at high risk for GDM (pre-pregnancy BMI $30 \mathrm{~kg} / \mathrm{m}^{2}$ or more and/or have a history of prior GDM) and (ii) in early pregnancy ( $<20$ weeks of gestation). In addition, participants had to have sufficient Finnish language skills to answer questionnaires and to understand the given lifestyle counselling.

Exclusion criteria were age under 18, continuous medication influencing glucose metabolism (such as oral corticosteroids or metformin), multiple pregnancy, physical disability, current substance abuse and severe psychiatric disorder. All participants gave informed consent at enrolment and the Ethics Committees of HUH (14 September 2006, Dnro 300/E9/06) and SKCH (11 September 2008, Dnro M06/08) approved the study.

The study visits took place three times during pregnancy (on average at 13, 23 and 35 weeks of gestation) and at 6 weeks, 6 months and 12 months after delivery.

The detailed design and methods of the intervention have been presented previously. ${ }^{27}$ In summary, participants in the intervention arm received structured but individually tailored dietary and physical activity counselling from the study nurses during the study visits. In addition, the intervention included a two-hour group nutrition counselling session given by a registered dietitian in early pregnancy. Dietary counselling in the intervention group was based on Nordic Nutrition Recommendations at the time. The recommendation for physical activity was a minimum of 150 min of moderate intensity physical activity per week. Participants in the usual care arm received similar information leaflets on healthy diet and exercise as provided in public antenatal care.

GDM was defined as one or more pathological plasma glucose value in $2 \mathrm{~h}$ oral 75 -g oral glucose tolerance test (OGTT) the thresholds being for fP-gluc $\geq 5.3$, one-hour $\mathrm{P}$-gluc $\geq 10.0$ and two-hour P-gluc $\geq 8.6 \mathrm{mmol} / \mathrm{l}$.

During pregnancy, all participants also attended routine communal antenatal clinic visits in primary health care centres. At the time of the study, this usual, common follow-up included 10-15 visits with a nurse and $2-3$ visits with a physician. Following the national GDM guidelines, diet treatment and self-monitoring of blood glucose were routinely initiated by antenatal clinics if GDM was diagnosed. In case of repeated fasting glucose of $\geq 5.5 \mathrm{mmol} / \mathrm{l}$ or a one-hour postprandial value of $\geq 7.8 \mathrm{mmol} / \mathrm{l}$, the antenatal clinic referred the woman to a maternity hospital for further counselling by a diabetes nurse, and to an obstetrician for an assessment regarding need of medication.

The HRQoL of the participants was assessed with the 15D. It is a validated, generic self-administered instrument and can be used both as a profile and as a single utility score measure for adults (over 16 years) ${ }^{28}$ As the $15 \mathrm{D}$ produces a single-index score, it can potentially be used as a measure of treatment effectiveness in cost-utility analyses. The 15D has been used in several patient groups, ${ }^{29}$ also among pregnant women. . $11,20,22,30,31^{2}$

The $15 \mathrm{D}$ questionnaire includes 15 dimensions: moving, seeing, hearing, breathing, sleeping, eating, speech, excretion, usual activities, mental function, discomfort and symptoms, depression, distress, vitality and sexual activity.

For each dimension, the respondent chooses a value between 1 and 5 to describe her current state of health (best level $=1$, worst level =5). These original values are transformed into a single-index score ranging from 0 to 1 , based on the multi-attribute utility theory. This total 15D score represents the subject's HRQoL with value 1 describing the best possible situation (no problems on any dimension) and 0 the worst (equivalent to being dead). ${ }^{28}$

We have tested $15 \mathrm{D}$-instrument in a general pregnant population earlier and demonstrated that it is responsive to HRQoL changes women experience during pregnancy and able to differentiate important dimensions that are affected also during normal pregnancy. $^{7}$

\section{Statistical methods}

The characteristics of the study groups are presented as means with standard deviations or as medians with interquartile range or as counts with percentages. Statistical comparison between the groups was performed by $t$-test, permutation test, Mann-Whitney test or Chi-square test, when appropriate. Repeated measures for $15 \mathrm{D}$ were analyzed using a generalized estimating equations (GEE) model with the unstructured correlation structure. GEEs were developed as an extension of the general linear model (e.g. OLS regression analysis) to analyze longitudinal and other correlated 
Table 1 Baseline demographic and clinical characteristics of the participants in the intervention and in the control groups

\begin{tabular}{|c|c|c|c|}
\hline & $\begin{array}{l}\text { Control } \\
N=186\end{array}$ & $\begin{array}{l}\text { Intervention } \\
N=192\end{array}$ & $P$-value \\
\hline Age (years), mean (SD) & $32.6(4.5)$ & $32.2(4.5)$ & 0.38 \\
\hline Body weight baseline $(\mathrm{kg})$, mean $(\mathrm{SD})$ & $90.1(17.6)$ & $90.5(16.9)$ & 0.86 \\
\hline Body mass index $(\mathrm{BMI})\left(\mathrm{kg} / \mathrm{m}^{2}\right)$, mean (SD) & $32.6(5.8)$ & $32.5(5.6)$ & 0.86 \\
\hline Years of education, mean (SD) & $14.5(2.0)$ & $14.4(2.1)$ & 0.52 \\
\hline Previous deliveries, $n(\%)$ & & & 0.54 \\
\hline None & $75(40)$ & 75 (39) & \\
\hline 1 & 72 (39) & $66(34)$ & \\
\hline 2 & $27(15)$ & $32(17)$ & \\
\hline 3 & $12(6)$ & $19(10)$ & \\
\hline Prior GDM, $n(\%)$ & $71(38)$ & $76(40)$ & 0.78 \\
\hline \multicolumn{4}{|l|}{ Blood pressure, mean (SD) } \\
\hline Systolic $(\mathrm{mmHg})$ & $121(13)$ & $123(13)$ & 0.079 \\
\hline Diastolic (mmHg) & $77(9)$ & $78(9)$ & 0.20 \\
\hline Fasting plasma glucose (mmol/l), mean (SD) & $5.08(0.39)$ & $5.06(0.43)$ & 0.64 \\
\hline Smoking, $n(\%)$ & $9(5)$ & $8(4)$ & 0.75 \\
\hline No alcohol use, $n(\%)$ & $176(95)$ & $185(96)$ & 0.42 \\
\hline Physical activity at baseline (min/week), median (IQR) & $60(30,140)$ & $60(30,120)$ & 0.91 \\
\hline Baseline OGTT pathological/performed (\%) & $64 / 180(36.7)$ & $69 / 188(37)$ & 0.82 \\
\hline
\end{tabular}

$\mathrm{IQR}$, inter quartile range.

data. GEE models take into account the correlation between repeated measurements in the same subject; models do not require complete data and can be fit even when individuals do not have observations at all time points. ${ }^{32}$ Effects in the models were group, time and the group-time interaction. All analyses were performed using STATA 15.0 (StataCorp LP, College Station, TX).

\section{Results}

A total of 492 of women recruited in early pregnancy were eligible and randomized to the RADIEL-study, after miscarriages and lost to follow-up 470 women completed the study, 239 of them in the intervention and 231 in the control group. This analysis includes those 378 participants that returned the $15 \mathrm{D}$ questionnaires at baseline and at least at one other time point, regardless of their OGTT results in the early pregnancy. Of the women included in this analysis, 192 received lifestyle counselling and 186 usual care.

The participants' baseline demographic and clinical characters are presented in table 1. Women of the intervention group did not differ from those of the control group regarding any of the variables assessed. In this population, the cumulative incidence of GDM was similar in the intervention and control groups (45.7 vs. $44.5 \%)$. The $15 \mathrm{D}$ scores of all pregnant women decreased similarly towards the third trimester with no significant difference between the intervention and the control group (adjusted for age and prepregnancy BMI) (figure 1). There were no differences between the $15 \mathrm{D}$-scores of women who were diagnosed with GDM during the pregnancy and those who were not (figure 2). The mean 15D-scores of women in the intervention and control group were also similar despite their GDM-status (figure 3).

\section{Discussion}

In this intervention study focussing on all the high-risk women allocated in the RADIEL-study in early pregnancy (including women with early GDM) we did not see any significant positive or negative differences in HRQoL between the intervention and the control group in this studied timeline.

In a previous Finnish lifestyle intervention study, ${ }^{30}$ women who had at least one risk-factor for GDM and were physically active reported higher $15 \mathrm{D}$-scores at the end of pregnancy compared to inactive women but regarding the change in $15 \mathrm{D}$ scores, the intervention was ineffective when compared to usual care. ${ }^{20}$ In that cluster-randomized study, the participants were not as obese as

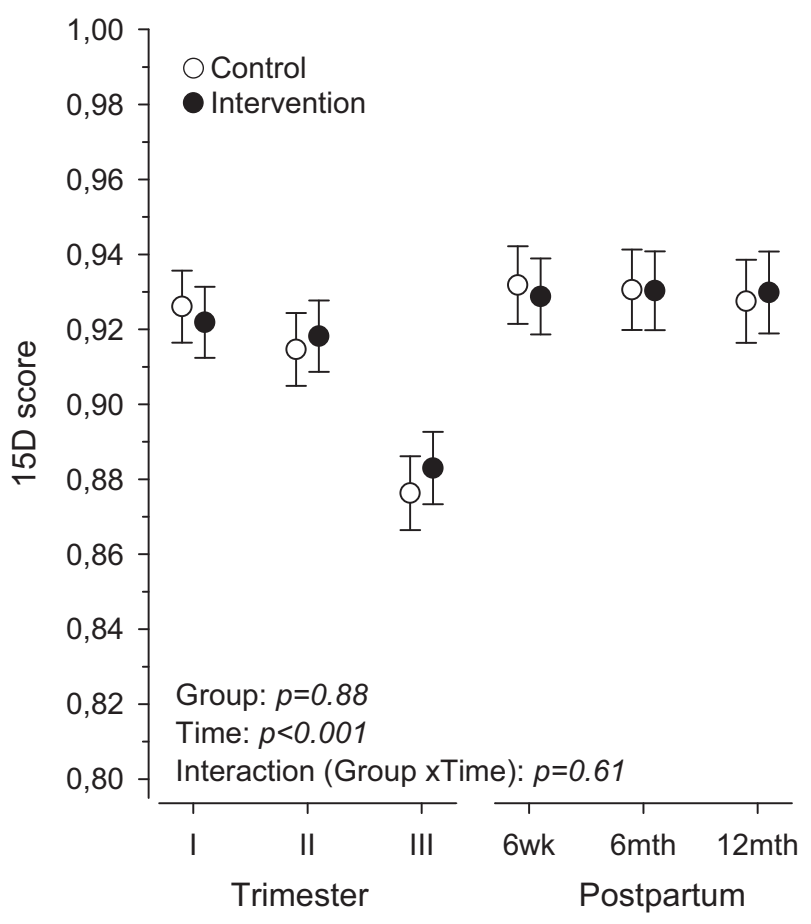

Figure 1 Mean 15D scores of intervention and control groups at different time points (adjusted for age and pre-pregnancy BMI). Missing values (Control, Intervention): II trim7, 7; III trim 19, 19; 6 wk postpartum 34, 32; 6 mth postpartum 47,48 and $12 \mathrm{mth}$ postpartum 66,69 . wk, week; mth, month

those in our study as their mean BMI was $\sim 26 \mathrm{~kg} / \mathrm{m}^{2}$, compared to $32 \mathrm{~kg} / \mathrm{m}^{2}$ in our study. ${ }^{31}$ There is evidence that obesity impairs the HRQoL of women, regardless of pregnancy and also independent of pregnancy-related complications. ${ }^{7}$ The participants of our study remained obese throughout their pregnancy and it can be speculated what the effect of this ongoing obesity on HRQoL was in our study group.

Our study population represents a true high-risk population for GDM as the cumulative incidence of GDM during the study was over $40 \%$ in the whole study group.

In line with most previous studies, ${ }^{9-11}$ we found no correlation between the GDM-status and HRQoL at any stage of pregnancy. 


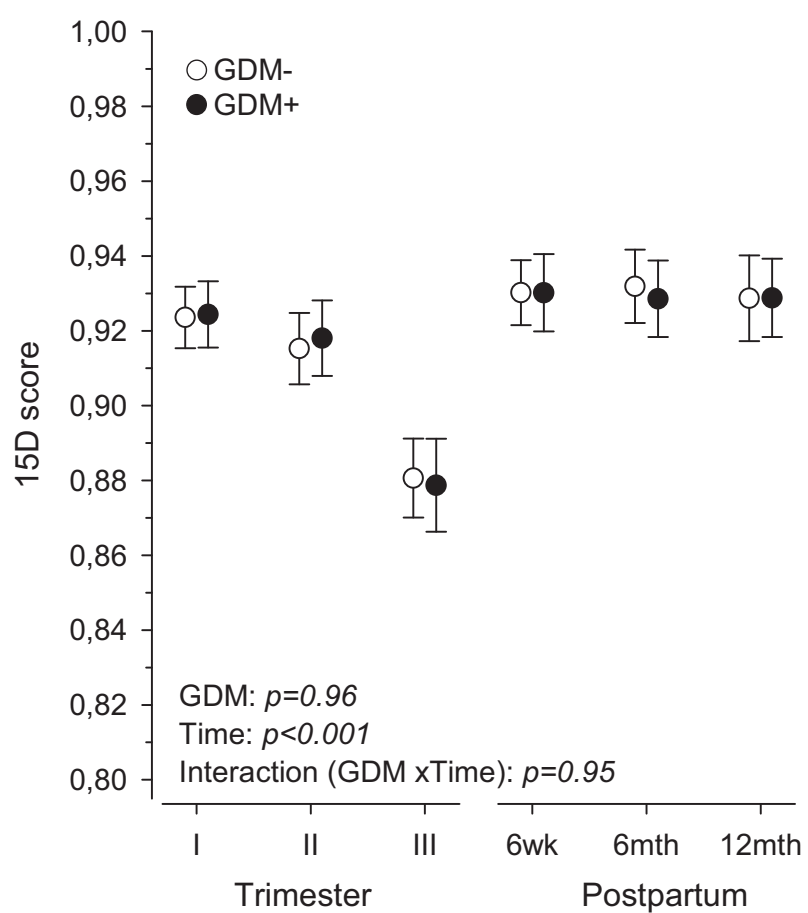

Figure 2 Mean 15D scores of participants with (GDM+) and without (GDM-) gestational diabetes at different time points, adjusted for age and pre-pregnancy BMI. Missing values (GDM-, GDM+): II trim8, 6; III trim 22, 15; 6 wk postpartum 36, 29; 6 mth postpartum 47,47 and 12 mth postpartum 71, 63. wk, week; mth, month

This observation seems logical, as GDM itself does not usually produce significant somatic symptoms.

Depressive symptoms during pregnancy can affect the HRQoL negatively $^{33}$ There have been studies suggesting that there is an increased risk for depressive symptoms among women that are at risk for or have been diagnosed with GDM. ${ }^{34}$ It is also worth noticing, that depression and GDM share some common socioeconomic risk-factors. ${ }^{35}$ In our study population, which consisted mostly of caucasian women with a higher educational status, the incidence of depressive symptoms in early pregnancy was similar to that in the general pregnant population. ${ }^{36}$

It is possible that the whole cohort was motivated to healthy lifestyle choices during their pregnancy, and the study group thus represented a more motivated and co-operative sample than the common high-risk population. Another possibility is that the women in the intervention group did not adopt the intervention into their daily practices as hoped. Also, our intervention was quite minimal (visits every 3 months) in addition to standard communal care (10-15 visits during pregnancy), and it can be speculated that our intervention was not intensive enough.

It is believed that during pregnancy women are quite receptive to healthy lifestyle incentives. Barriers for making healthy choices may be internal (physical or psychological) or external (work, family, time, environment). The advice provided should be personalized and address also these individual reasons and beliefs. ${ }^{37}$

The RADIEL-study intervention protocol was designed to be feasible and easy to implement in the primary-care setting. The visits to study nurse included individually modifiable dietary and physical activity counselling from trained study nurses. ${ }^{27}$ It remains unclear, how deeply these individual, internal and external barriers could be addressed during these visits. Also, the level and intensity of physical activity of the participants before the intervention, level of activity remain unclear. Despite encouragement to regular physical activity, some of the participants may have had fears and

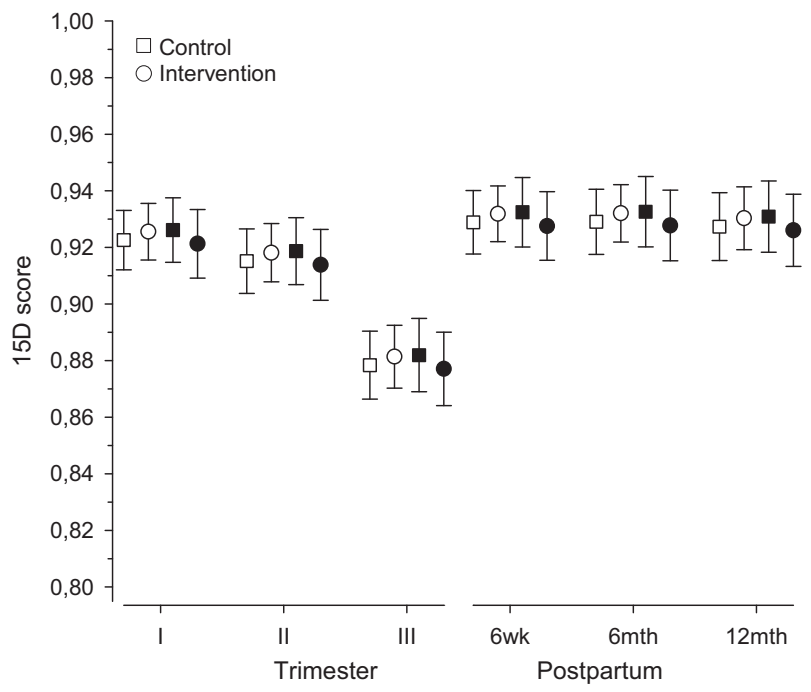

Figure 3 Mean 15D scores of participants in intervention and control groups with (black) and without (white) gestational diabetes at different time points (adjusted for age and prepregnancy BMI)

misconceptions concerning, e.g. the safety of physical activity during pregnancy. ${ }^{37}$

The contents, interventions, and effects of routine communal antenatal clinic visits in our study also remain unclear. Since all recruited women belonged to high GDM risk group and shared commonly acknowledged risk-factors for unfavourable pregnancy outcomes, they received more support for healthy lifestyle choices from maternity clinics. Also, the routine treatment for GDM given by communal antenatal clinics acts as a strong intervention for those $40 \%$ of women that were given the diagnosis of GDM during their pregnancy potentially confounding the effect of RADIEL-intervention. It is difficult to accurately differentiate effect of the given intervention from treatment of GDM in public healthcare. It is also not possible to evaluate the effect of those social and environmental factors which could have contributed to the opportunity to truly make behavioural changes during this study.

Motivating people to lifestyle changes is challenging and interventions that aim to change health-related behaviours tend to have only modest effects. ${ }^{38}$ People are on the cognitive level usually well aware of the positive effects of healthy lifestyle. Even in such a possibly motivated study group as ours, the results of the intervention advocating healthier lifestyle remained modest or minor. Continuously exploring, in true co-operation with the women at high risk for the GDM, the ways of implementing the existing knowledge into the daily practices of women and their families is essential.

\section{Acknowledgements}

We would like to thank Hannu Kautiainen MSc for the statistical analyses. We would also like to warmly thank women attending the RADIEL-study and the RADIEL-study nurses.

\section{Disclaimer}

The funders have not had any role in designing or conducting the study; in the collection, management, analysis or interpretation of the data; in the preparation, review or approval of the manuscript and in the decision to submit the manuscript for publication. 


\section{Funding}

The RADIEL-study has received funds from the Ahokas Foundation, the Finnish Foundation for Cardiovascular Disease, Special State Subsidy for Health Science Research of Helsinki University Central Hospital, Samfundet Folkhälsan, The Finnish Diabetes Research Foundation, the State Provincial Office of Southern Finland and the Social Insurance Institution of Finland.

Conflicts of interest: None declared.

\section{Key points}

- A combined diet and physical activity intervention did not prove to have a positive effect on HRQoL of women at high risk for GDM even when the intervention was started in early pregnancy.

- Diagnosis of GDM among high-risk women does not seem to affect the HRQoL of women during pregnancy or up to 12 months postpartum.

- Motivating women at high risk for GDM to implement healthier lifestyle choices into their daily practice may be difficult.

\section{References}

1 Simmons D. Prevention of gestational diabetes mellitus: where are we now? Diabetes Obes Metab 2015;17:824-34.

2 Shepherd E, Gomersall JC, Tieu J, et al. Combined diet and exercise interventions for preventing gestational diabetes mellitus. Cochrane Database Syst Rev 2017;11:CD010443.

3 Koivusalo B, Rönö K, Klemetti R, et al. Gestational Diabetes Mellitus Can Be Prevented by Lifestyle Intervention. The Finnish Gestational Diabetes Prevention Study (RADIEL). Diabetes Care 2016;39:24-30.

4 World Health Organisation. WHOQOL: Measuring Quality of Life 1997. 1997. Available at: http://www.who.int/mental_health/media/68.pdf (3 October 2017, date last accessed).

5 Preedy VR, Watson RR. Handbook of Disease Burdens and Quality of Life Measures. New York: Springer, 2010.

6 Drummond MF, Sculpher MJ, Torrance GW, et al. Methods for the Economic Evaluation of Health Care Programmes, 3rd edn. Oxford: Oxford University Press, 2005.

7 Drummond MF, Schwartz JS, Jönsson B. Key principles for the improved conduct of health technology assessments for resource allocation decisions. Int J Technol Assess Health Care 2008;24:244-58.

8 Sahrakorpi N, Koivusalo SB, Stach-Lempinen B, et al. "The Burden of Pregnancy"; heavier for the heaviest? The changes in Health Related Quality of Life (HRQoL) assessed by the $15 \mathrm{D}$ instrument during pregnancy and postpartum in different body mass index groups: a longitudinal survey. Acta Obstet Gynecol Scand 2017;96:352-8.

9 Amador N, Juarez JM, Guízar JM, Linares M. Quality of life in obese pregnant women: a longitudinal study. Am J Obstet Gynecol 2008;198:2031-5.

10 Kim C, Brawarsky P, Jackson RA, et al. Changes in health status experienced by women with gestational diabetes and pregnancy-induced hypertensive disorders. J Women's Health (Larchmt) 2005;14:729-36.

11 Danyliv A, Gillespie P, O’Neill C, et al. Health related quality of life two to five years after gestational diabetes mellitus: cross-sectional comparative study in the ATLANTIC DIP cohort. BMC Pregnancy Childbirth 2015;15:274.

12 Halkoaho A, Kavilo M, Pietilä AM, et al. Does gestational diabetes affect women's health-related quality of life after delivery? Eur J Obstet Gynecol Reprod Biol 2010;148:40-3.

13 Dalfra MG, Nicolucci A, Bisson T, et al. Quality of life in pregnancy and postpartum: a study in diabetic patients. Qual Life Res 2012;21:291-8.

14 Marchetti D, Carrozzino D, Fraticelli F, et al. Quality of Life in Women with Gestational Diabetes Mellitus: A Systematic Review. J Diabetes Res 2017;2017:1.

15 Tendais I, Figueiredo B, Mota J, Conde A. Physical activity, health-related quality of life and depression during pregnancy. Cad Saúde Pública 2011;27:219-28.
16 Haakstad L, Torset B, Bø $\mathrm{K}$. What is the effect of regular group exercise on maternal psychological outcomes and common pregnancy complaints? An assessor blinded RCT. Midwifery 2016;32:81-6.

17 Gustafsson MK, Stafne SN, Romundstad PR, et al. The effects of an exercise programme during pregnancy on health-related quality of life in pregnant women: a Norwegian randomized controlled trial. BJOG 2016;123:1152-60.

18 Petrov Fieril K, Glantz A, Fagevik Olsen M. The efficacy of moderate-to-vigorous resistance exercise during pregnancy: a randomized controlled trial. Acta Obstet Gynecol Scand 2015;94:35-42.

19 Nascimento SL, Surita FG, Parpinelli MA, et al. The effect of an antenatal physical exercise programme on maternal/perinatal outcomes and quality of life in overweight and obese pregnant women: a randomized clinical trial. BJOG 2011;118:1455-63.

20 Kolu P, Raitanen J, Rissanen P, Luoto R. Cost-effectiveness of lifestyle counselling as primary prevention of gestational diabetes mellitus: findings from a clusterrandomised trial. PLoS One 2013;8:e56392.

21 Oostdam N, Bosmans J, Wouters MG, et al. Cost-effectiveness of an exercise program during pregnancy to prevent gestational diabetes: Results of an economic evaluation alongside a randomised controlled trial. BMC Pregnancy Childbirth 2012;12:64.

22 Kolu P, Raitanen J, Puhkala J, et al. Effectiveness and Cost-Effectiveness of a ClusterRandomized Prenatal Lifestyle Counseling Trial: A Seven-Year Follow-Up. PLoS One 2016;11:e0167759.

23 Tieu J, Shepherd E, Middleton P, Crowther CA. Dietary advice interventions in pregnancy for preventing gestational diabetes mellitus. Cochrane Database Syst Rev 2017;1:CD006674.

24 McCarthy EA, Walker SP, Ugoni A, et al. Self-weighing and simple dietary advice for overweight and obese pregnant women to reduce obstetric complications without impact on quality of life: a randomised controlled trial. BJOG 2016;123:965-73.

25 Brown J, Ceysens G, Boulvain M. Exercise for pregnant women with gestational diabetes for improving maternal and fetal outcomes. Cochrane Database Syst Rev 2017;6:CD012202.

26 Brown J, Alwan NA, West J, et al. Lifestyle interventions for the treatment of women with gestational diabetes. Cochrane Database Syst Rev 2017;5:CD011970.

27 Rönö K, Stach-Lempinen B, Klemetti MM. Prevention of gestational diabetes through lifestyle intervention: study design and methods of a Finnish randomized controlled multicenter trial (RADIEL). BMC Pregnancy Childbirth 2014;14:1-11.

28 Sintonen $\mathrm{H}$. The 15D instrument of health-related quality of life: properties and applications. Ann Med 2001;33:328-36.

29 15D-instrument. 2018. Available at: http://15d-instrument.net/publications/ (3 October 2017, date last accessed).

30 Kolu P, Raitanen J, Luoto R. Physical activity and health-related quality of life during pregnancy: a secondary analysis of a cluster-randomized trial. Matern Child Health J 2014;18:2098-105.

31 Luoto R, Kinnunen TI, Aittasalo M, et al. Primary prevention of gestational diabetes mellitus and large-for- gestational-age newborns by lifestyle counseling: a clusterrandomized controlled trial. PLoS Med 2011;8:e1001036.

32 Liang KY, Zeger SL. Longitudinal data analysis using generalized linear models. Biometrika 1986;73:13-22.

33 Li J, Mao J, Du Y, et al. Health related quality of life among pregnant women with and without depression in Hubei, China. Matern Child Health J 2012;16:1355-63.

34 Hinkle SN, Buck Louis GN, Rawal S, et al. A longitudinal study of depression and gestational diabetes in pregnancy and the postpartum period. Diabetologia 2016;59:2594-602.

35 Walmer R, Huynh J, Wenger J, et al. Health disorders subsequent to gestational dianbetes mellitus differ by race/ethnicity. Depress Anxiety 2015;32:774-82.

36 Engberg E, Stach-Lempinen B, Sahrakorpi N, et al. A cross-sectional study of antenatal depressive symptoms at high risk for gestational diabetes mellitus. J Psychosom Res 2015;79:646-50.

37 Weir Z, Bush J, Robson SC, et al. Physical activity in pregnancy: a qualitative study of the beliefs of overweight and obese pregnant women. BMC Pregnancy Childbirth 2010;10:18.

38 Davis R, Campbell R, Hildon Z, et al. Theories of behaviour and behaviour change across the social and behavioural sciences: a scoping review. Health Psychol Rev 2015;9:323-44 